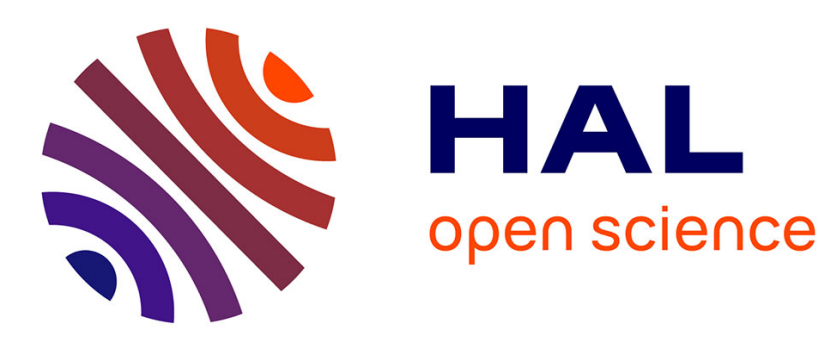

\title{
Submillimeter-wave spectroscopy of and interstellar search for thioacetaldehyde
}

Laurent Margulès, V. V. Ilyushin, Brett A. Mcguire, A. Belloche, R. A.

Motiyenko, A. J. Remijan, E. A. Alekseev, O. Dorovskaya, J. -C. Guillemin

\section{- To cite this version:}

Laurent Margulès, V. V. Ilyushin, Brett A. Mcguire, A. Belloche, R. A. Motiyenko, et al.. Submillimeter-wave spectroscopy of and interstellar search for thioacetaldehyde. Journal of Molecular Spectroscopy, 2020, 371, pp.111304. 10.1016/j.jms.2020.111304 . hal-02928605

\section{HAL Id: hal-02928605 https://hal.science/hal-02928605}

Submitted on 10 Sep 2020

HAL is a multi-disciplinary open access archive for the deposit and dissemination of scientific research documents, whether they are published or not. The documents may come from teaching and research institutions in France or abroad, or from public or private research centers.
L'archive ouverte pluridisciplinaire HAL, est destinée au dépôt et à la diffusion de documents scientifiques de niveau recherche, publiés ou non, émanant des établissements d'enseignement et de recherche français ou étrangers, des laboratoires publics ou privés. 


\title{
Submillimeter-wave spectroscopy of and interstellar search for thioacetaldehyde
}

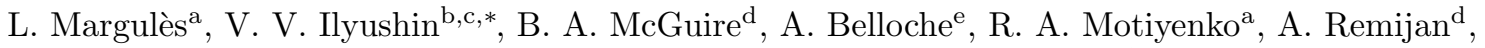 \\ E. A. Alekseev ${ }^{\text {b,c }}$, O. Dorovskaya ${ }^{\text {b }}$, J.-C. Guillemin ${ }^{f}$ \\ ${ }^{a}$ Univ. Lille, CNRS, UMR 8523 - PhLAM - Physique des Lasers Atomes et Molécules, F-59000 Lille, France \\ ${ }^{b}$ Institute of Radio Astronomy of NASU, 4, Mystetstv St., Kharkiv, 61002, Ukraine \\ ${ }^{c}$ Quantum Radiophysics Department, V.N. Karazin Kharkiv National University, Svobody Square 4, 61022, Kharkiv, Ukraine \\ ${ }^{d}$ National Radio Astronomy Observatory, Charlottesville, VA 22903, USA \\ ${ }^{e}$ Max-Planck-Institut für Radioastronomie, Auf dem Hügel 69, 53121 Bonn, Germany \\ ${ }^{f}$ Univ. Rennes, Ecole Nationale Supérieure de Chimie de Rennes, CNRS, ISCR - UMR 6226, F-35000 Rennes, France
}

\begin{abstract}
We present a new study of the millimeter and submillimeter wave spectra of the thioacetaldehyde molecule, $\mathrm{CH}_{3} \mathrm{CHS}$, a sulfur-bearing analog of the ubiquitous interstellar molecule acetaldehyde $\left(\mathrm{CH}_{3} \mathrm{CHO}\right)$. Here, we present a laboratory investigation aimed at determining accurate spectroscopic parameters for $\mathrm{CH}_{3} \mathrm{CHS}$ to enable astronomical searches for this molecule using radio telescope arrays at millimeter and submillimeter wavelengths. New laboratory measurements have been carried out between 150 and $660 \mathrm{GHz}$ using the spectrometer of PhLAM in Lille (France). Rotational transitions up to $J=60$ were assigned in the ground, first and second excited torsional states of thioacetaldehyde and fit using the RAM Hamiltonian model. The final fit includes 62 parameters to give an overall weighted root-mean-square deviation of 0.9. On the basis of our spectroscopic results, $\mathrm{CH}_{3} \mathrm{CHS}$ was searched for, but not detected, in data from the Atacama Large Millimeter/submillimeter Array (ALMA) between $84 \mathrm{GHz}$ and $114 \mathrm{GHz}$ toward the hot molecular core Sgr B2(N2). The non-detection implies that thioacetaldehyde is at least 90 times less abundant than acetaldehyde in this source. We also searched for but found no evidence of thioacetaldehyde in a number of prestellar and protostellar sources targeted by the Astrochemical Surveys at IRAM (ASAI) Large Program in nearby low-mass star forming regions.
\end{abstract}

Keywords: Microwave spectrum, torsional large amplitude motion, thioacetaldehyde, ISM

\section{Introduction}

More than 200 molecules have thus far been detected in the interstellar medium (ISM) [1. Of these, $\sim 70$ and $\sim 20$ are oxygen-bearing and sulfur-bearing chemical compounds, respectively. Sulfur is the tenth most abundant element in the galaxy and to date, almost all sulfur compounds detected in the ISM have the corresponding oxygen derivatives also observed in the ISM. Existing problems with the systematic understanding of interstellar sulfur chemistry [2, 3, 4, 5] have motivated recent searches for new sulfurbearing interstellar molecules. To that purpose a number of studies of sulfur-bearing analogs, not yet detected in the ISM, of well known oxygen-bearing interstellar molecules have been performed in recent years: thioacetic acid $\left(\mathrm{CH}_{3} \mathrm{COSH}\right)$ [], thioacetamide $\left(\mathrm{CH}_{3} \mathrm{CSNH}_{2}\right)$ ]7, dimethylsulfide $\left(\left(\mathrm{CH}_{3}\right)_{2} \mathrm{~S}\right)$ [8], O- and Sthiomethylformates $\left(\mathrm{CH}_{3} \mathrm{SCHO}, \mathrm{CH}_{3} \mathrm{OCHS}\right)$ 9. In this work, we present the results of a new spectroscopic study for the thioacetaldehyde molecule $\left(\mathrm{CH}_{3} \mathrm{CHS}\right)$. With the corresponding oxygen derivative, acetaldehyde [10] as well as the unsubstituted derivative, thioformaldehyde, [11] being already detected in the ISM,

\footnotetext{
* Corresponding author

Email address: ilyushin@rian.kharkov.ua (V. V. Ilyushin)
} 
thioacetaldehyde is a reasonable candidate for detection in the ISM. Except for the tentatively detected ethanethiol, 12 which contains 9 atoms, all the detected sulfur compounds have less than 7 atoms. If detected, thioacetaldehyde would therefore be one of the largest sulfur-bearing species in the ISM.

Several prior spectroscopic and synthetic studies have been devoted to thioacetaldehyde. As early as 1974, Kroto et al. 13 reported the photoelectron and microwave spectrum of this compound generated by vacuum flash pyrolysis (VFP) of the corresponding trimer: 1,3,5-trimethyl s-trithiane. The microwave spectrum showed evidence of hindered internal rotation [13] and it was detailed a few years later together 20 with a study of eight isotopic variants of thioacetaldehyde [14. The measurements were done up to 40 $\mathrm{GHz}$ and transitions up to $J=35$ in the ground torsional state were assigned [14. Information on dipole moment as well as methyl top internal rotation barrier heights was also obtained. Using the same approach, visible, UV spectra and phosphorescence excitation and emission spectra were observed [15, 16. The VFP of alkyl thiosulfinates [17] or thiocyanohydrines [18 was also used to characterize or to trap the formed thioacetaldehyde. The photolysis of 2-methylthietane and 2,4-dimethylthietane in argon matrices allowed recording matrix infrared spectra [19]. Thioacetaldehyde was also prepared by matrix photolysis of ethanesulfenyl chloride or thiirane and by VFP of allylethylsulfide [20] and generated by photolysis of phenacylethylsulfide or by thermolysis of thiabicycloheptene 21.

In this paper, we present the results of our study of the torsion-rotational spectrum of thioacetaldehyde. The aim of the study is to obtain accurate spectroscopic parameters of thioacetaldehyde to support astronomical observations by radio telescope arrays, in particular at millimeter and submillimeter wavelengths. The new measurements were carried out between $150 \mathrm{GHz}$ and $660 \mathrm{GHz}$ and analyzed using the so-called rho-axis method (RAM). The analysis of the spectrum was performed up to $J=60$ and up to the second excited torsional state, and a fit within experimental error was obtained. Predictions based on our final fit

35 are used to search for $\mathrm{CH}_{3} \mathrm{CHS}$ with the Atacama Large Millimeter/submillimeter Array (ALMA) toward the hot molecular core Sgr B2(N2) as well as in a number of observational datasets from the Astrochemical Surveys at IRAM (ASAI) Large Program.

The rest of the paper is organized as follows. In Section 2 we give in brief experimental details for the new measurements used in this work. In Section 3 we describe the assignment process and our fitting results.

40 Section 4 describes our astronomical observations and the results of our search for thioacetaldehyde. Section 5 summarizes the results obtained in this study.

\section{Experimental Section}

\subsection{Synthesis}

Thioacetaldehyde was synthesized by flash vacuum thermolysis of ethyl-2-propynyl sulfide 22 at $750^{\circ} \mathrm{C}$ 45 under 0.1 mbar. A stoichiometric amount of allene, a compound without permanent dipolar moment, was formed during the reaction. The gaseous flow was directly introduced in the cell of the spectrometer.

\subsection{Lille - submillimeter spectra}

The measurements in the frequency range under investigation were performed using the Lille spectrometer 23, equipped with a fast-scan mode [24. The frequency ranges 150-330, and 400-660 GHz were covered with various active and passive frequency multipliers from VDI Inc. and an Agilent synthesizer $(12.5-18.25 \mathrm{GHz})$ was used as the reference source of radiation. The absorption cell was a stainless-steel tube (6 cm diameter, $220 \mathrm{~cm}$ long). During measurements, the sample was at a pressure of about $10 \mathrm{~Pa}$ and at room temperature, the linewidth was limited by Doppler broadening. Estimated uncertainties for measured line frequencies are $30 \mathrm{kHz}, 50 \mathrm{kHz}, 100 \mathrm{kHz}$, and $200 \mathrm{kHz}$ depending on the observed S/N and the frequency range.

\section{Spectroscopic analysis and results}

The so-called RAM torsion-rotation Hamiltonian used in this work is based on the works [25], [26], and 27]. We employed the RAM36 (rho-axis-method for 3 and 6-fold barriers) code that realizes the RAM 
approach for the molecules with the $C_{3 v}$ top attached to a molecular frame of $C_{s}$ or $C_{2 v}$ symmetry and having 3- or 6-fold barrier to internal rotation respectively [28, 29]. This code was successfully applied to the oxygen-bearing analog of thioacetaldehyde, acetaldehyde [30, which has comparable and rather large coupling between internal and overall rotations ( $\rho=0.33$ for $\mathrm{CH}_{3} \mathrm{CHO}, \rho=0.26$ for $\mathrm{CH}_{3} \mathrm{CHS}$ ) as well as $V_{3}$ value $\left(V_{3}=407.6 \mathrm{~cm}^{-1}\right.$ for $\mathrm{CH}_{3} \mathrm{CHO}, V_{3}=556.6 \mathrm{~cm}^{-1}$ for $\left.\mathrm{CH}_{3} \mathrm{CHS}\right)$. A general expression for the RAM Hamiltonian implemented in this code as well as further details of the used theoretical approach may be found in Refs. [28, 29]. The fits for thioacetaldehyde were performed with 21 torsional basis functions in the first diagonalization step and 9 torsional basis functions in the second diagonalization step.

We started our analysis from the results of [14, and as the first step we have refit the dataset of [14 with the RAM36 program [28, 29]. The initial predictions obtained from this refit were good enough for starting assignments in the $150-330 \mathrm{GHz}$ range. Further assignments were performed in a usual bootstrap manner, with numerous cycles of refinement of the parameter set while the new data were gradually added. It should be noted that initially we aimed at studying only the ground torsional state with a number of low $K$ series from the first excited torsional state included for stabilizing the torsional part of the Hamiltonian, since we were mainly interested in reliable predictions for astronomical use. But it appeared that for this dataset we were not able to get a fit within experimental error (the weighted root mean square (rms) deviation was about 4.5). One of our assumptions was that the obtained solution corresponded to some local minima and in order to try to get out of it we decided to add the second excited torsional state to our analysis. Following several cycles of adding new $v_{t}=1$ and $v_{t}=2$ data to the fit and refinement of the parameter set confirmed correctness of our assumption and finally let us get a fit within experimental error for $v_{t}=0,1,2$ dataset.

The final dataset of the current study includes both our new measurements and the data from the literature [14] (6 lines out of 93 were excluded from Ref. 14 dataset because of large obs.-cal. values). The dataset contains $8333 A$ - and $E$-type transitions with $J \leq 60$ and $K_{a} \leq 25$ that due to blending correspond to 6908 measured lines. The final weighted rms deviation was 0.9 with all groups of data fitted within their measurement uncertainties. The final set of molecular parameters is presented in Table 1. It contains 62 parameters, which are distributed between the $n_{o p}=2,4,6,8,10$ orders as $7,21,25,6,3$, respectively. This ${ }_{85}$ is consistent with the total numbers of determinable parameters of 7, 22, 50, 95, and 161 for those orders, as calculated from the differences between the total number of symmetry-allowed Hamiltonian terms of order $n_{o p}$ and the number of symmetry-allowed contact transformation terms of order $n_{o p}-1$ [31]. There were no convergence problems observed for the final fit.

Table 1: Fitted parameters of the RAM Hamiltonian for the thioacetaldehyde molecule.

\begin{tabular}{lllc}
\hline \hline$n_{t r}{ }^{\mathrm{a}}$ & Parameter $^{\mathrm{b}}$ & Operator $^{\mathrm{c}}$ & Value $^{\mathrm{d}}$ \\
\hline $2_{20}$ & $F$ & $p_{\alpha}^{2}$ & $6.809672(26)$ \\
$2_{20}$ & $V_{3}$ & $\frac{1}{2}(1-\cos 3 \alpha)$ & $556.6140(24)$ \\
$2_{11}$ & $\rho$ & $J_{z} p_{\alpha}$ & $0.26038433(72)$ \\
$2_{02}$ & $A_{R A M}$ & $J_{z}^{2}$ & $1.61784741(70)$ \\
$2_{02}$ & $B_{R A M}$ & $J_{x}^{2}$ & $0.19945055(20)$ \\
$2_{02}$ & $C_{R A M}$ & $J_{y}^{2}$ & $0.176923079(33)$ \\
$2_{02}$ & $D_{z x}$ & $\left\{J_{z}, J_{x}\right\}$ & $-0.1047031(14)$ \\
$4_{40}$ & $F_{m}$ & $p_{\alpha}^{4}$ & $-0.39768(66) \times 10^{-3}$ \\
$4_{40}$ & $V_{6}$ & $\frac{1}{2}(1-\cos 6 \alpha)$ & $-22.7413(28)$ \\
$4_{31}$ & $\rho_{m}$ & $J_{z} p_{\alpha}^{3}$ & $-0.66956(86) \times 10^{-3}$ \\
$4_{22}$ & $F_{J}$ & $J^{2} p_{\alpha}^{2}$ & $0.18497(48) \times 10^{-5}$ \\
$4_{22}$ & $F_{K}$ & $J_{z}^{2} p_{\alpha}^{2}$ & $-0.80501(43) \times 10^{-3}$ \\
$4_{22}$ & $F_{z x}$ & $\frac{1}{2} p_{\alpha}^{2}\left\{J_{z}, J_{x}\right\}$ & $0.14856(53) \times 10^{-4}$ \\
$4_{22}$ & $V_{3 J}$ & $J^{2}(1-\cos 3 \alpha)$ & $0.253960(94) \times 10^{-3}$ \\
$4_{22}$ & $V_{3 K}$ & $J_{z}^{2}(1-\cos 3 \alpha)$ & $-0.192064(64) \times 10^{-1}$ \\
$4_{22}$ & $V_{3 z x}$ & $\frac{1}{2}(1-\cos 3 \alpha)\left\{J_{z}, J_{x}\right\}$ & $0.17186(19) \times 10^{-2}$ \\
$4_{22}$ & $V_{3 x y}$ & $\left(J_{x}^{2}-J_{y}^{2}\right)(1-\cos 3 \alpha)$ & $0.127485(86) \times 10^{-3}$ \\
\hline
\end{tabular}


Table 1: continued.

\begin{tabular}{|c|c|c|c|}
\hline$n$ ntr $^{\mathrm{a}}$ & Parameter $^{\mathrm{b}}$ & Operator $^{\mathrm{c}}$ & Value $^{\mathrm{d}}$ \\
\hline $4_{22}$ & $D_{3 z y}$ & $\frac{1}{2} \sin 3 \alpha\left\{J_{z}, J_{y}\right\}$ & $-0.8237(15) \times 10^{-2}$ \\
\hline $4_{22}$ & $D_{3 x y}$ & $\frac{1}{2} \sin 3 \alpha\left\{J_{x}, J_{y}\right\}$ & $0.2399(38) \times 10^{-4}$ \\
\hline $4_{13}$ & $\rho_{J}$ & $J^{2} J_{z} p_{\alpha}$ & $0.85246(25) \times 10^{-5}$ \\
\hline $4_{13}$ & $\rho_{K}$ & $J_{z}^{3} p_{\alpha}$ & $-0.46829(14) \times 10^{-3}$ \\
\hline $4_{13}$ & $\rho_{z x}$ & $\frac{1}{2} p_{\alpha}\left\{J_{z}^{2}, J_{x}\right\}$ & $0.8797(57) \times 10^{-5}$ \\
\hline $4_{04}$ & $D_{z x J}$ & $\frac{1}{2}\left\{J_{z}, J_{x}\right\} J^{2}$ & $0.75397(24) \times 10^{-6}$ \\
\hline $4_{04}$ & $\Delta_{J}$ & $-J^{4}$ & $0.1210501(89) \times 10^{-6}$ \\
\hline $4_{04}$ & $\Delta_{J K}$ & $-J^{2} J_{z}^{2}$ & $-0.447150(52) \times 10^{-5}$ \\
\hline $4_{04}$ & $\Delta_{K}$ & $-J_{z}^{4}$ & $0.108273(22) \times 10^{-3}$ \\
\hline $4_{04}$ & $\delta_{J}$ & $-2 J^{2}\left(J_{x}^{2}-J_{y}^{2}\right)$ & $0.287961(44) \times 10^{-7}$ \\
\hline $4_{04}$ & $\delta_{K}$ & $-\left\{J_{z}^{2},\left(J_{x}^{2}-J_{y}^{2}\right)\right\}$ & $-0.688(11) \times 10^{-7}$ \\
\hline $6_{60}$ & $V_{9}$ & $\frac{1}{2}(1-\cos 9 \alpha)$ & $0.3553(22)$ \\
\hline $6_{42}$ & $F_{m J}$ & $J^{2} p_{\alpha}^{4}$ & $0.317(10) \times 10^{-8}$ \\
\hline $6_{42}$ & $V_{6 J}$ & $J^{2}(1-\cos 6 \alpha)$ & $0.37406(66) \times 10^{-4}$ \\
\hline 642 & $V_{6 K}$ & $J_{z}^{2}(1-\cos 6 \alpha)$ & $-0.5154(70) \times 10^{-3}$ \\
\hline $6_{42}$ & $D_{6 z y}$ & $\frac{1}{2} \sin 6 \alpha\left\{J_{z}, J_{y}\right\}$ & $0.4466(27) \times 10^{-3}$ \\
\hline $6_{33}$ & $\rho_{m K}$ & $J_{z}^{3} p_{\alpha}^{3}$ & $0.897(28) \times 10^{-7}$ \\
\hline 633 & $\rho_{m x y}$ & $\frac{1}{2} p_{\alpha}^{3}\left\{J_{z},\left(J_{x}^{2}-J_{y}^{2}\right)\right\}$ & $-0.1371(54) \times 10^{-8}$ \\
\hline 633 & $\rho_{3 x y}$ & $\frac{1}{2}\left\{J_{z}, J_{x}, J_{y}, p_{\alpha}, \sin 3 \alpha\right\}$ & $0.1617(41) \times 10^{-5}$ \\
\hline $6_{24}$ & $F_{J K}$ & $J^{2} J_{z}^{2} p_{\alpha}^{2}$ & $-0.3857(50) \times 10^{-8}$ \\
\hline $6_{24}$ & $F_{K K}$ & $J_{z}^{4} p_{\alpha}^{2}$ & $0.1421(19) \times 10^{-6}$ \\
\hline $6_{24}$ & $V_{3 J J}$ & $J^{4}(1-\cos 3 \alpha)$ & $-0.25678(69) \times 10^{-8}$ \\
\hline $6_{24}$ & $V_{3 K K}$ & $J_{z}^{4}(1-\cos 3 \alpha)$ & $0.2616(28) \times 10^{-5}$ \\
\hline $6_{24}$ & $V_{3 x y \mathrm{~J}}$ & $J^{2}(1-\cos 3 \alpha)\left(J_{x}^{2}-J_{y}^{2}\right)$ & $-0.12861(61) \times 10^{-8}$ \\
\hline $6_{24}$ & $V_{3 x 2 y 2}$ & $\frac{1}{2} \cos 3 \alpha\left\{J_{x}^{2}, J_{y}^{2}\right\}$ & $0.2712(46) \times 10^{-8}$ \\
\hline $6_{24}$ & $V_{3 z x K}$ & $\frac{1}{2}(1-\cos 3 \alpha)\left\{J_{z}^{3}, J_{x}\right\}$ & $-0.9077(71) \times 10^{-6}$ \\
\hline $6_{24}$ & $D_{3 x y K}$ & $\frac{1}{2} \sin 3 \alpha\left\{J_{z}^{2}, J_{x}, J_{y}\right\}$ & $0.2067(73) \times 10^{-6}$ \\
\hline $6_{15}$ & $\rho_{J K}$ & $J^{2} J_{z}^{3} p_{\alpha}$ & $-0.3414(21) \times 10^{-8}$ \\
\hline 615 & $\rho_{K K}$ & $J_{z}^{5} p_{\alpha}$ & $0.7595(49) \times 10^{-7}$ \\
\hline $6_{06}$ & $D_{z x J K}$ & $\frac{1}{2} J^{2}\left\{J_{z}^{3}, J_{x}\right\}$ & $0.389(17) \times 10^{-10}$ \\
\hline $6_{06}$ & $\Phi_{J}$ & $J^{6}$ & $0.19952(55) \times 10^{-12}$ \\
\hline $6_{06}$ & $\Phi_{J K}$ & $J^{4} J_{z}^{2}$ & $-0.825(19) \times 10^{-11}$ \\
\hline $6_{06}$ & $\Phi_{K J}$ & $J^{2} J_{z}^{4}$ & $-0.8072(37) \times 10^{-9}$ \\
\hline $6_{06}$ & $\Phi_{K}$ & $J_{z}^{6}$ & $0.14083(59) \times 10^{-7}$ \\
\hline 606 & $\phi_{J}$ & $2 J^{4}\left(J_{x}^{2}-J_{y}^{2}\right)$ & $0.8980(27) \times 10^{-13}$ \\
\hline $6_{06}$ & $\phi_{J K}$ & $J^{2}\left\{J_{z}^{2},\left(J_{x}^{2}-J_{y}^{2}\right)\right\}$ & $-0.2730(65) \times 10^{-11}$ \\
\hline $8_{62}$ & $V_{9 K}$ & $J_{z}^{2}(1-\cos 9 \alpha)$ & $-0.699(33) \times 10^{-4}$ \\
\hline $8_{53}$ & $\rho_{6 x y}$ & $\frac{1}{2}\left\{J_{z}, J_{x}, J_{y}, p_{\alpha}, \sin 6 \alpha\right\}$ & $-0.405(13) \times 10^{-6}$ \\
\hline $8_{26}$ & $F_{K K K}$ & $J_{z}^{6} p_{\alpha}^{2}$ & $0.1347(37) \times 10^{-10}$ \\
\hline $8_{26}$ & $V_{3 J J J}$ & $J^{6}(1-\cos 3 \alpha)$ & $0.825(27) \times 10^{-14}$ \\
\hline $8_{08}$ & $L_{J K}$ & $J^{4} J_{z}^{4}$ & $0.1473(71) \times 10^{-14}$ \\
\hline $8_{08}$ & $L_{K K J}$ & $J^{2} J_{z}^{6}$ & $-0.458(21) \times 10^{-13}$ \\
\hline $10_{64}$ & $V_{9 J J}$ & $J^{4}(1-\cos 9 \alpha)$ & $-0.4033(48) \times 10^{-10}$ \\
\hline $10_{64}$ & $D_{9 x y K}$ & $\frac{1}{2} \sin 9 \alpha\left\{J_{z}^{2}, J_{x}, J_{y}\right\}$ & $-0.793(34) \times 10^{-7}$ \\
\hline $10_{46}$ & $V_{6 K K K}$ & $J_{z}^{6}(1-\cos 6 \alpha)$ & $-0.1877(97) \times 10^{-9}$ \\
\hline
\end{tabular}


Table 1: continued.

\begin{tabular}{l}
\hline \hline$n t r^{\mathrm{a}}$ Parameter $^{\mathrm{b}}$ Operator $^{\mathrm{c}}$ \\
\hline${ }^{\mathrm{a}} n=t+r$, where $n$ is the total order of the operator, $t$ is the order of the torsional \\
part and $r$ is the order of the rotational part, respectively. \\
b Parameter nomenclature based on the subscript procedures of ref. [32. \\
c $\{A, B\}=A B+B A,\{A, B, C\}=A B C+C B A$, etc. The product of the operator \\
in the third column of a given row and the parameter in the second column of \\
that row gives the term actually used in the torsion-rotation Hamiltonian of the \\
program, except for $F, \rho$ and $A_{R A M}$, which occur in the Hamiltonian in the form \\
$F\left(p_{\alpha}-\rho J_{z}\right)^{2}+A_{R A M} J_{z}^{2}$. \\
d All values are in cm ${ }^{-1}$ (except $\rho$ which is unitless). Statistical uncertainties are \\
shown as one standard uncertainty in the units of the last two digits.
\end{tabular}

On the basis of the parameters of our final fit, we calculated a list of transitions for astronomical use. This list includes information on transition quantum numbers, transition frequencies, calculated uncertainties, lower state energies, and transition strengths. The predictions are made up to $700 \mathrm{GHz}$ for $v_{t} \leq 2$ and $J \leq 60$, and we limit our predictions to the transitions with calculated uncertainties lower than $0.1 \mathrm{MHz}$. The RAM36 code labels energy levels by free rotor quantum number $m$, overall rotational angular momentum quantum number $J$, and $K_{a}, K_{c}$ numbers. Table 2 summarizes relations between torsional $v_{t}$ and free rotor $m$ quantum numbers, also giving energies of the $\mathrm{A} / \mathrm{E}$ torsional substates. Lower state energies in Table 2 (as well as in the list of transitions for astronomical use) are given referenced to the $J=0 A$-type $v_{t}=0$ level. This level was calculated to be $82.4851 \mathrm{~cm}^{-1}$ above the bottom of the torsional potential well. Thus $v_{t}=2 \mathrm{E}$ substate is approximately $168 \mathrm{~cm}^{-1}$ below the top of the barrier. The line strengths have been calculated using the values $\mu_{a}=2.26 \mathrm{D}$ and $\mu_{b}=0.56 \mathrm{D}$ [14], which were transformed to the RAM axis system of the current study. In addition, we provide the rotation-torsion part of the partition function $Q_{r t}(\mathrm{~T})$ of thioacetaldehyde calculated via direct summation over the rotational-torsion states. The states up to $J=100$ and $v_{t}=8$ were taken into account in $Q_{r t}(\mathrm{~T})$ calculation. The predictions, partition function as well as line list of the data set treated in our study are available in the Supplementary material associated with this article.

Table 2: Energies of the $J=0$ levels for $v_{t}=0,1,2$ states of thioacetaldehyde (in $\mathrm{cm}^{-1}$ ).

\begin{tabular}{lcrcr}
\hline \hline$v_{t}$ & A-type & $m$ & E-type & $m$ \\
\hline 0 & 0.0 & 0 & 0.0098 & 1 \\
1 & 159.5637 & -3 & 159.2535 & -2 \\
2 & 301.8678 & 3 & 305.8351 & 4 \\
\hline
\end{tabular}

\section{Radioastronomical Observations}

\subsection{Search toward Sgr B2(N2) with ALMA}

We used the spectroscopic predictions derived in this work for thioacetaldehyde to search for this molecule toward the hot molecular core Sgr B2(N2). We used the imaging spectral line survey EMoCA (Exploring Molecular Complexity with ALMA) conducted toward Sgr B2(N) with the Atacama Large Millimeter/submillimeter Array (ALMA) in its observing cycles 0 and 1. Sgr B2(N) is a protocluster forming high-mass stars located in the giant molecular cloud Sgr B2, at about 100 pc in projection from the Galactic center and a distance of $8.2 \mathrm{kpc} 33$. Details about the survey and the data calibration and imaging were reported in ref. 34. In short, the survey was performed with a median angular resolution of $1.6^{\prime \prime}$ and a phase center located at $(\alpha, \delta)_{\mathrm{J} 2000}=\left(17^{\mathrm{h}} 47^{\mathrm{m}} 19 \mathrm{~s} 87,-28^{\circ} 22^{\prime} 16^{\prime \prime} \cdot 0\right)$. The survey covers the frequency range from 84.1 to $114.4 \mathrm{GHz}$ with a spectral resolution of $488 \mathrm{kHz}$, which corresponds to 1.7 to $1.3 \mathrm{~km} \mathrm{~s}$ over this frequency range. Here we analyze the spectrum at the peak position of Sgr B2(N2) located at $(\alpha, \delta)_{\mathrm{J} 2000}=\left(17^{\mathrm{h}} 47^{\mathrm{m}} 19 \mathrm{~s} 86,-28^{\circ} 22^{\prime} 13^{\prime \prime} 4\right)$. The spectrum is modeled with the Weeds software 35] under the 

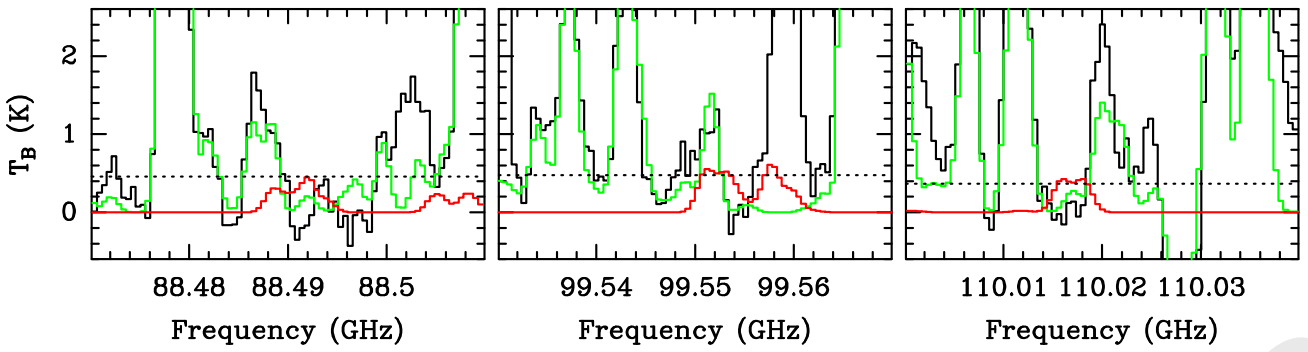

Figure 1: Synthetic LTE spectrum of thioacetaldehyde (in red) used to derive the upper limit to its column density, overlaid on the ALMA spectrum of Sgr B2(N2) (in black) and the synthetic spectrum that contains the contributions of all the species (but not thioacetaldehyde) that we have identified so far in this source on the basis of the EMoCA survey (in green). The dotted line in each panel indicates the $3 \sigma$ noise level. Other transitions of thioacetaldehyde that are expected to be weaker than $3 \sigma$ and/or heavily contaminated by other species are not shown.

assumption of local thermodynamic equilibrium (LTE), which is adequate given the high densities at the scales probed with EMoCA in Sgr B2(N2) $\left(>1 \times 10^{7} \mathrm{~cm}^{-3}\right.$, see [36]).

We did not detect any transition of thioacetaldehyde in the EMoCA spectrum of Sgr B2(N2). Figure 1 displays three frequency ranges that illustrate the non-detection. The LTE synthetic spectrum used to derive the upper limit to the column density of thioacetaldehyde is displayed in red and was computed assuming the same parameters (temperature, emission size, linewidth, and velocity offset with respect to the systemic velocity) as the ones we derived for acetaldehyde with the EMoCA survey [37. These parameters are listed in Table 3, along with the parameters derived previously for methanol and methyl mercaptan in Sgr B2(N2) using the same survey [38. We find that thioacetaldehyde is at least $\sim 90$ times less abundant than acetaldehyde in Sgr B2(N2). For comparison, methyl mercaptan is about 120 times less abundant than methanol in Sgr B2(N2). The non-detection of thioacetaldehyde is thus not surprising if the abundance ratio of oxygen-bearing to sulfur-bearing molecules is the same for these two families of organic molecules in this source.

Table 3: Parameters of our best-fit LTE model of methanol, methyl mercaptan, acetaldehyde ${ }^{\mathrm{a}}$, and column density upper limit for thioacetaldehyde, toward Sgr B2(N2) on the basis of the EMoCA survey.

\begin{tabular}{|c|c|c|c|c|c|c|c|c|c|}
\hline Molecule & Status $^{\mathrm{b}}$ & $N_{\text {det }}{ }^{c}$ & $\begin{array}{l}\text { Size }^{\mathrm{d}} \\
\left({ }^{\prime \prime}\right)\end{array}$ & $\begin{array}{l}T_{\text {rot }} \mathrm{e} \\
(\mathrm{K})\end{array}$ & $\begin{array}{c}N^{\mathrm{f}} \\
\left(\mathrm{cm}^{-2}\right)\end{array}$ & $F_{\text {vib }} \mathrm{g}$ & $\begin{array}{c}\Delta V^{\mathrm{h}} \\
\left(\mathrm{km} \mathrm{s}^{-1}\right)\end{array}$ & $\begin{array}{c}V_{\text {off }^{\mathrm{i}}} \\
\left(\mathrm{km} \mathrm{s}^{-1}\right)\end{array}$ & $\frac{N_{\text {ref }}}{N} \mathrm{j}$ \\
\hline $\mathrm{CH}_{3} \mathrm{OH}, v=0^{\star}$ & 4 & 41 & 1.4 & 160 & $4.0(19)$ & 1.00 & 5.4 & -0.5 & 1 \\
\hline $\mathrm{CH}_{3} \mathrm{SH}, v=0$ & d & 12 & 1.4 & 180 & $3.4(17)$ & 1.00 & 5.4 & -0.5 & 118 \\
\hline $\mathrm{CH}_{3} \mathrm{CHO}, v=0^{\star}$ & $\mathrm{d}$ & 19 & 1.1 & 160 & $5.3(17)$ & 1.08 & 5.6 & 0.0 & 1 \\
\hline $\mathrm{CH}_{3} \mathrm{CHS}, v=0$ & $\mathrm{n}$ & 0 & 1.1 & 160 & $<6.2(15)$ & 1.03 & 5.6 & 0.0 & $>86$ \\
\hline
\end{tabular}

a The parameters for methanol and methyl mercaptan were published in ref. [38, and for acetaldehyde in ref. [37.

$\mathrm{b}$ d: detection, n: non-detection.

c Number of detected lines (conservative estimate, see Sect. 3 of ref. 34]). One line of a given species may mean a group of transitions of that species that are blended together.

d Source diameter $(F W H M)$.

e Rotational temperature.

f Total column density of the molecule. $x(y)$ means $x \times 10^{y}$.

$\mathrm{g}$ Correction factor that was applied to the column density to account for the contribution of vibrationally excited states, in the cases where this contribution was not included in the partition function of the spectroscopic predictions.

${ }^{\mathrm{h}}$ Linewidth $(F W H M)$.

i Velocity offset with respect to the assumed systemic velocity of Sgr B2(N2), $V_{\text {sys }}=74 \mathrm{~km} \mathrm{~s}^{-1}$.

j Column density ratio, with $N_{\text {ref }}$ the column density of the previous reference species marked with a $\star$.

\subsection{Search using Astrochemical Surveys at IRAM Large Program}

We have searched for thioacetaldehyde in a number of observational datasets from the Astrochemical Surveys at IRAM Large Program, which are publicly available and have been previously published elsewhere. 
The ASAI spectra cover a range of source types from cold, dark clouds to Class 0/1 protostars and shocked outflows, with observational details available in Ref. 39. Because the observational details of these sources are described elsewhere; only brief overview is provided here. For each source, because these are nondetections, we have had to make assumptions about the physical conditions in which thioacetaldehyde might be found. For all sources considered in this subsection, these parameters were chosen to match those used in a similar analysis of cyanoketene [40] (since many of these sources do not have acetaldehyde detections in them). These parameters and the literature sources they were obtained from are provided in Table 4

Table 4: Source parameters assumed for thioacetaldehyde in each of the ASAI sets of observations.

\begin{tabular}{|c|c|c|c|c|c|c|c|}
\hline Source & Telescope & $\begin{array}{l}\theta_{s}^{a} \\
\left({ }^{\prime \prime}\right)\end{array}$ & $\begin{array}{l}T_{b g}^{b} \\
(\mathrm{~K})\end{array}$ & $\begin{array}{c}\Delta V \\
\left(\mathrm{~km} \mathrm{~s}^{-1}\right)\end{array}$ & $\begin{array}{c}T_{b}^{\dagger} \\
(\mathrm{mK})\end{array}$ & $\begin{array}{l}T_{e x}^{b} \\
(\mathrm{~K})\end{array}$ & Refs. \\
\hline Barnard 1 & IRAM & - & 2.7 & 0.8 & 2.5 & 10 & 41,42 \\
\hline IRAS 4A & IRAM & - & 2.7 & 5.0 & 2.3 & 21 & 41,43 \\
\hline L1157B1 & IRAM & - & 2.7 & 8.0 & 1.4 & 60 & 44 \\
\hline L1157mm & IRAM & - & 2.7 & 3.0 & 2.8 & 60 & 44 \\
\hline L1448R2 & IRAM & - & 2.7 & 8.0 & 2.4 & 60 & 45 \\
\hline L1527 & IRAM & - & 2.7 & 0.5 & 1.9 & 12 & 45,46 \\
\hline L1544 & IRAM & - & 2.7 & 0.5 & 2.3 & 10 & 47,48 \\
\hline SVS13A & IRAM & 0.3 & 2.7 & 3.0 & 7.5 & 80 & 41,43 \\
\hline TMC1 & IRAM & - & 2.7 & 0.3 & 6.5 & 7 & 49,50 \\
\hline
\end{tabular}

${ }^{a}$ Except where noted, the source is assumed to fill the beam.

${ }^{b} T_{b g}$ is the background temperature. $T_{e x}$ is the excitation temperature.

$\dagger$ Taken either as the $3 \sigma$ RMS noise level at the location of the target line, or for line confusion limited spectra, the reported $3 \sigma$ RMS noise of the observations.

We find no evidence of thioacetaldehyde in any of our studied sources. We derive upper limits to the column density in each source using the formalisms outlined in Ref. [51, which assume the molecules are well described by a single excitation temperature and include corrections for optical depth. Frequencies, energy levels, degeneracies, and line strengths were obtained from the laboratory spectroscopy described in this work. The partition function, which includes a correction for the lowest torsional vibrational mode of the molecule, is given in Supplementary Material associated with this article. In warmer source $(T>100 \mathrm{~K})$, vibrational corrections from higher lying modes must also be considered. To that end, we have carried out quantum chemical calculations using Gaussian 09 [52] at the wB97XD/6-311++G(d,p) level of theory and basis set to determine the harmonic frequencies of thioacetaldehyde. The harmonic frequencies are also given in the Supplementary Material.

For each source, we simulated a spectrum using the physical conditions for the source and the line parameters measured in this work. Then, the $3 \sigma$ upper limit to the column density was derived, using the line that gave the most rigorous constraint (i.e. the line that would have the highest signal-to-noise ratio in the event of a detection). All lines used to derive upper limits to the column density belong to the ground torsional state of thioacetaldehyde. These lines are provided in Table 5 along with the resulting upper limits.

\section{Conclusion}

A new study of the rotational spectrum of thioacetaldehyde $\mathrm{CH}_{3} \mathrm{CHS}$ was carried out in the frequency range of $150-660 \mathrm{GHz}$ in order to provide accurate predictions for astronomical searches. The rotational transitions of the ground, first and second excited torsional states were assigned up to $J=60$ and fit using

160 a RAM Hamiltonian within experimental accuracy. The overall weighted rms deviation of the global fit of 6908 line frequencies of $\mathrm{CH}_{3} \mathrm{CHS}$ is 0.9 , indicating that we have an appropriate set of parameters to provide reliable predictions for astronomical observations. Unfortunately, our first attempt to search for $\mathrm{CH}_{3} \mathrm{CHS}$ with ALMA data between $84 \mathrm{GHz}$ and $114 \mathrm{GHz}$ toward the hot molecular core Sgr B2(N2) was not successful. The non-detection implies that thioacetaldehyde is at least 90 times less abundant than 
Table 5: $3 \sigma$ upper limits to thioacetaldehyde and the line parameters used to calculate them in each of the ASAI sets of observations.

\begin{tabular}{|c|c|c|c|c|c|c|c|c|c|}
\hline Source & $\begin{array}{c}\text { Frequency } \\
(\mathrm{MHz})\end{array}$ & $\begin{array}{c}\text { Transition } \\
\left(S: J_{K_{a}, K_{c}}^{\prime}-J_{K_{a}, K_{c}}^{\prime \prime}\right)\end{array}$ & $\begin{array}{l}E_{u} \\
(\mathrm{~K}) \\
\end{array}$ & $\begin{array}{c}S_{i j} \mu^{2} \\
\left(\text { Debye }^{2}\right) \\
\end{array}$ & $\bar{Q}\left(Q_{t r}, Q_{v i b}\right)^{a}$ & $\begin{array}{c}N_{T} \\
\left(\mathrm{~cm}^{-2}\right)\end{array}$ & $\begin{array}{l}\mathrm{N}\left(\mathrm{H}_{2}\right) \\
\left(\mathrm{cm}^{-2}\right)\end{array}$ & $\mathrm{X}_{\mathrm{H}_{2}}$ & Refs. \\
\hline Barnard 1 & 101408.2 & $E: 9_{1,8}-8_{1,7}$ & 22.4 & 45.4 & $267(267,1.00)$ & $\leq 6.9 \times 10^{11}$ & $1.5 \times 10^{23}$ & $\leq 6 \times 10^{-12}$ & 42 \\
\hline IRAS $4 \mathrm{~A}$ & 108228.9 & $A: 10_{1,10}-9_{1,9}$ & 30.7 & 50.6 & $861(861,1.00)$ & $\leq 2.5 \times 10^{12}$ & $3.7 \times 10^{23}$ & $\leq 6 \times 10^{-12}$ & 42 \\
\hline $\mathrm{L} 1157 \mathrm{~B} 1$ & 108228.9 & $A: 10_{1,10}-9_{1,9}$ & 30.7 & 50.6 & $861(861,1.00)$ & $\leq 3.6 \times 10^{12}$ & $1 \times 10^{21}$ & $\leq 3 \times 10^{-9}$ & 42 \\
\hline $\mathrm{L} 1157 \mathrm{~mm}$ & 108228.9 & $A: 10_{1,10}-9_{1,9}$ & 30.7 & 50.6 & $861(861,1.00)$ & $\leq 4.2 \times 10^{12}$ & $6 \times 10^{21}$ & $\leq 6 \times 10^{-10}$ & 42 \\
\hline L1448R2 & 97429.9 & $A: 9_{1,9}-8_{1,8}$ & 25.5 & 45.4 & $4161(4161,1.00)$ & $\leq 7.2 \times 10^{12}$ & $3.5 \times 10^{23}$ & $\leq 2 \times 10^{-11}$ & 45 \\
\hline L1527 & 86623.7 & $A: 8_{1,8}-7_{1,7}$ & 20.8 & 40.2 & $364(364,1.00)$ & $\leq 3.0 \times 10^{11}$ & $2.8 \times 10^{22}$ & $\leq 1 \times 10^{-11}$ & 45 \\
\hline L1544 & 99110.2 & $E: 9_{0,9}-8_{0,8}$ & 23.8 & 46.0 & $267(267,1.00)$ & $\leq 2.9 \times 10^{11}$ & $5 \times 10^{21}$ & $\leq 6 \times 10^{-11}$ & 53 \\
\hline SVS13A & 255531.7 & A: $23_{3,20}-22_{3,19}$ & 165.4 & 116.0 & $6666(6661,1.00)$ & $\leq 1.0 \times 10^{16}$ & $3 \times 10^{24}$ & $\leq 3 \times 10^{-9}$ & 54 \\
\hline TMC1 & 135082.5 & $E: 12_{1,11}-11_{1,10}$ & 44.3 & 60.9 & $129(129,1.00)$ & $\leq 1.0 \times 10^{13}$ & $1 \times 10^{22}$ & $\leq 9 \times 10^{-10}$ & 42 \\
\hline
\end{tabular}

${ }^{a}$ Calculated at the excitation temperature assumed for the source. See Table 4 protostellar sources targeted by the ASAI Large Program. Based on our results, frequency predictions for astrophysical use were produced up to $700 \mathrm{GHz}$. The predictions, as well as data set treated in our study, are available as supplementary material to this article.

\section{Acknowledgements}

This work was supported by the Programme National "Physique et Chimie du Milieu Interstellaire" (PCMI) of CNRS/INSU with INC/INP co-funded by CEA and CNES. Support for B.A.M. was provided by NASA through Hubble Fellowship grant \#HST-HF2-51396 awarded by the Space Telescope Science Institute, which is operated by the Association of Universities for Research in Astronomy, Inc., for NASA, under contract NAS5-26555. The National Radio Astronomy Observatory is a facility of the National the Centre National d'Etudes Spatiales (CNES) for a grant. The research in Kharkiv was carried out under support of the Volkswagen foundation. The assistance of the Science and Technology Center in the Ukraine is acknowledged (STCU partner project P686). This paper makes use of the following ALMA data: ADS/JAO.ALMA\#2011.0.00017.S, ADS/JAO.ALMA\#2012.1.00012.S. ALMA is a partnership of ESO (representing its member states), NSF (USA), and NINS (Japan), together with NRC (Canada), NSC and ASIAA (Taiwan), and KASI (Republic of Korea), in cooperation with the Republic of Chile. The Joint ALMA Observatory is operated by ESO, AUI/NRAO, and NAOJ. The interferometric data are available in the ALMA archive at https://almascience.eso.org/aq/. Part of this work has been carried out within the Collaborative Research Centre 956, sub-project B3, funded by the Deutsche Forschungsgemeinschaft (DFG) - project ID 184018867.

\section{References}

[1] B. A. McGuire, 2018 Census of Interstellar, Circumstellar, Extragalactic, Protoplanetary Disk, and Exoplanetary Molecules, The Astrophysical Journal Supplement Series 239 (2) (2018) 17.

[2] R. Martín-Doménech, I. Jiménez-Serra, G. M. Muñoz Caro, H. S. P. Müller, A. Occhiogrosso, L. Testi, P. M. Woods, S. Viti, The sulfur depletion problem: upper limits on the $\mathrm{H}_{2} \mathrm{~S}_{2}, \mathrm{HS} \cdot{ }_{2}$, and $\mathrm{S}_{2}$ gas-phase abundances toward the low-mass warm core IRAS 16293-2422, Astronomy and Astrophysics 585 (2016) A112.

[3] T. H. G. Vidal, V. Wakelam, A new look at sulphur chemistry in hot cores and corinos, Monthly Notices of the Royal Astronomical Society 474 (4) (2018) 5575-5587.

[4] C. Vastel, D. Quénard, R. Le Gal, V. Wakelam, A. Andrianasolo, P. Caselli, T. Vidal, C. Ceccarelli, B. Lefloch, R. Bachiller, Sulphur chemistry in the L1544 pre-stellar core, Monthly Notices of the Royal Astronomical Society 478 (4) (2018) 55145532.

[5] J. C. Laas, P. Caselli, Modeling sulfur depletion in interstellar clouds, Astronomy and Astrophysics 624 (2019) A108.

[6] C. J. Smith, A. K. Huff, H. Zhang, Y. Mo, K. R. Leopold, A strong dependence of the CH3 internal rotation barrier on conformation in thioacetic acid: Microwave measurements and an energy decomposition analysis, The Journal of Chemical Physics 150 (13) (2019) 134302. 
[7] A. Maris, C. Calabrese, L. B. Favero, L. Evangelisti, I. Usabiaga, S. Mariotti, C. Codella, L. Podio, N. Balucani, C. Ceccarelli, B. LeFloch, S. Melandri, Laboratory Measurements and Astronomical Search for Thioacetamide, ACS Earth and Space Chemistry 3 (8) (2019) 1537-1549.

8] V. Ilyushin, I. Armieieva, O. Dorovskaya, P. M, I. Krapivin, E. Alekseev, L. Margulès, R. Motiyenko, F. Kwabia Tchana, A. Jabri, L. Manceron, E. S. Bekhtereva, S. Bauerecker, C. Maul, Microwave and FIR spectroscopy of dimethylsulfide in the ground, first and second excited torsional states, Journal of Molecular Structure 1200 (2020) 127114.

[9] A. Jabri, R. A. Motiyenko, L. Margules, J.-C. Guillemin, E. A. Alekseev, I. Kleiner, B. Tercero, J. Cernicharo, Millimeter wave spectrum of the two monosulfur derivatives of methyl formate: S- and O-methyl thioformate, in the ground and the first excited torsional states, 72 International Symposium on Molecular Spectroscopy, June 19-23, 20171200 (2017) id. WF07.

10] C. A. Gottlieb, Detection of acetaldehyde in Sagittarius, in: Molecules in the Galactic Environment, 1973, p. 181.

[11] M. Sinclair, N. Fourikis, J. Ribes, B. Robinson, R. Brown, P. Godfrey, Detection of interstellar thioformaldehyde, Australian Journal of Physics 26 (1) (1973) 85-92.

[12] L. Kolesniková, B. Tercero, J. Cernicharo, J. Alonso, A. Daly, B. Gordon, S. Shipman, Spectroscopic characterization and detection of Ethyl Mercaptan in Orion, The Astrophysical Journal Letters 784 (1) (2014) L7.

[13] H. Kroto, B. Landsberg, R. Suffolk, A. Vodden, The photoelectron and microwave spectra of the unstable species thioacetaldehyde, $\mathrm{CH}_{3} \mathrm{CHS}$, and thioacetone, $\left(\mathrm{CH}_{3}\right)_{2} \mathrm{CS}$, Chemical Physics Letters 29 (2) (1974) 265-269.

14] H. Kroto, B. Landsberg, The microwave spectrum, substitution structure, internal rotation barrier, and dipole moment of thioacetaldehyde, $\mathrm{CH}_{3}$ CHS, Journal of Molecular Spectroscopy 62 (3) (1976) 346-363.

220 [15] R. Judge, D. Moule, A. Bruno, R. Steer, Thioketone spectroscopy: An analysis of the lower electronic transitions in thioacetone and thioacetaldehyde, Chemical physics letters 102 (4) (1983) 385-389.

[16] R. Judge, D. Moule, A. Bruno, R. Steer, Thiocarbonyl spectroscopy: Methyl torsional vibrations and internal rotational barriers of thioacetaldehyde in its $\mathrm{a}^{3} \mathrm{~A}^{\prime} \mathrm{X}^{1} \mathrm{~A}^{\prime}$ states, The Journal of chemical physics 87 (1) (1987) 60-67.

[17] J. E. Baldwin, R. G. Lopez, The generation and trapping of thiobenzaldehyde and thioacetaldehyde, Journal of the Chemical Society, Chemical Communications (18) (1982) 1029-1030.

[18] A. Gaumont, L. Wazneh, J. Denis, Thiocyanohydrins, a new class of compounds, precursors of unstabilized thiocarbonyl derivatives., Tetrahedron 47 (27) (1991) 4927-4940.

[19] E. Suzuki, O. Watanabe, A. Happoya, E. Watari, Photolysis of 2-methylthietane and 2, 4-dimethylthietane in argon matrices: matrix infrared spectra of thioacetaldehyde, Vibrational spectroscopy 5 (3) (1993) 353-357.

20] G. Maier, U. Flögel, H. P. Reisenauer, B. H. Andes Jr, L. J. Schaad, HCl-Abspaltung aus Ethansulfenylchlorid und Chlordimethylsulfid, Chemische Berichte 124 (11) (1991) 2609-2612.

[21] E. Vedejs, S. Fields, Reaction of thioaldehydes with 5-alkoxyoxazoles. A route to 3-thiazolines, The Journal of Organic Chemistry 53 (20) (1988) 4663-4667.

[22] J. A. Varela, L. Castedo, M. Maestro, J. Mahía, C. Saá, Regiocontrolled One-Step Synthesis of 3,3'-Disubstituted 2,2'Bipyridine Ligands by Cobalt (I)-Catalyzed Cyclotrimerization, Chemistry-A European Journal 7 (23) (2001) 5203-5213.

[23] O. Zakharenko, R. A. Motiyenko, L. Margulès, T. R. Huet, Terahertz spectroscopy of deuterated formaldehyde using a frequency multiplication chain, Journal of Molecular Spectroscopy 317 (2015) 41-46.

[24] R. A. Motiyenko, I. A. Armieieva, L. Margulès, E. A. Alekseev, J.-C. Guillemin, Rotational spectroscopy of malononitrile and its corresponding monoisocyanide isomer, isocyanoacetonitrile, Astronomy and Astrophysics 623 (2019) A162.

25] B. Kirtman, Interactions between ordinary vibrations and hindered internal rotation. I. Rotational energies, The Journal of Chemical Physics 37 (11) (1962) 2516-2539.

[26] R. Lees, J. Baker, Torsion-vibration-rotation interactions in methanol. I. Millimeter wave spectrum, The Journal of Chemical Physics 48 (12) (1968) 5299-5318.

[27] E. Herbst, J. Messer, F. C. De Lucia, P. Helminger, A new analysis and additional measurements of the millimeter and submillimeter spectrum of methanol, Journal of Molecular Spectroscopy 108 (1) (1984) 42-57.

[28] V. V. Ilyushin, Z. Kisiel, L. Pszczólkowski, H. Mäder, J. T. Hougen, A new torsion-rotation fitting program for molecules with a sixfold barrier: Application to the microwave spectrum of toluene, Journal of Molecular Spectroscopy 259 (1) (2010) $26-38$.

[29] V. V. Ilyushin, C. P. Endres, F. Lewen, S. Schlemmer, B. J. Drouin, Submillimeter wave spectrum of acetic acid, Journal of Molecular Spectroscopy 290 (2013) 31-41.

[30] I. Smirnov, E. Alekseev, V. Ilyushin, L. Margulés, R. Motiyenko, B. Drouin, Spectroscopy of the ground, first and second excited torsional states of acetaldehyde from 0.05 to $1.6 \mathrm{THz}$, Journal of Molecular Spectroscopy 295 (2014) 44-50.

[31] K. Nakagawa, S. Tsunekawa, T. Kojima, Effective torsion-rotation Hamiltonian for methanol-type molecules, Journal of Molecular Spectroscopy 126 (2) (1987) 329-340.

[32] L.-H. Xu, J. Fisher, R. Lees, H. Shi, J. Hougen, J. Pearson, B. Drouin, G. Blake, R. Braakman, Torsion-rotation global analysis of the first three torsional states $\left(\nu_{t}=0,1,2\right)$ and terahertz database for methanol, Journal of Molecular Spectroscopy 251 (1-2) (2008) 305-313.

[33] M. J. Reid, K. M. Menten, A. Brunthaler, X. W. Zheng, T. M. Dame, Y. Xu, J. Li, N. Sakai, Y. Wu, K. Immer, B. Zhang, A. Sanna, L. Moscadelli, K. L. J. Rygl, A. Bartkiewicz, B. Hu, L. H. Quiroga-Nuñez, H. J. van Langevelde, Trigonometric parallaxes of high-mass star-forming regions: Our view of the milky way, The Astrophysical Journal 885 (2) (2019) 131.

[34] A. Belloche, H. Müller, R. Garrod, K. Menten, Exploring molecular complexity with ALMA (EMoCA): Deuterated complex organic molecules in Sagittarius B2 (N2), Astronomy and Astrophysics 587 (2016) A91.

[35] S. Maret, P. Hily-Blant, J. Pety, S. Bardeau, E. Reynier, Weeds: a CLASS extension for the analysis of millimeter and sub-millimeter spectral surveys, Astronomy and Astrophysics 526 (2011) A47.

[36] M. Bonfand, A. Belloche, R. T. Garrod, K. M. Menten, E. Willis, G. Stéphan, H. S. P. Müller, The complex chemistry of 
hot cores in Sgr B2(N): influence of cosmic-ray ionization and thermal history, Astronomy and Astrophysics 628 (2019) A27.

[37] M. Sanz-Novo, A. Belloche, J. L. Alonso, L. Kolesniková, R. T. Garrod, S. Mata, H. S. P. Müller, K. M. Menten, Y. Gong, Interstellar glycolamide: A comprehensive rotational study and an astronomical search in Sgr B2(N), Astronomy and Astrophysics submitted.

[38] H. S. Müller, A. Belloche, L.-H. Xu, R. M. Lees, R. T. Garrod, A. Walters, J. van Wijngaarden, F. Lewen, S. Schlemmer, K. M. Menten, Exploring molecular complexity with ALMA (EMoCA): Alkanethiols and alkanols in Sagittarius B2 (N2), Astronomy and Astrophysics 587 (2016) A92.

[39] B. Lefloch, R. Bachiller, C. Ceccarelli, J. Cernicharo, C. Codella, A. Fuente, C. Kahane, A. Lopez-Sepulcre, M. Tafalla, C. Vastel, E. Caux, M. González García, E. Bianchi, A. Gómez-Ruiz, J. Holdship, E. Mendoza, J. Ospina-Zamudio, L. Podio, D. Quenard, E. Roueff, N. Sakai, S. Viti, S. Yamamoto, K. Yoshida, C. Favre, T. Monfredini, H. M. QuitiánLara, N. Marcelino, H. M. Boechat-Roberty, S. Cabrit, Astrochemical evolution along star formation: overview of the IRAM Large Program ASAI, Monthly Notices of the Royal Astronomical Society 477 (4) (2018) 4792-4809.

[40] L. Margulès, B. A. McGuire, R. A. Motiyenko, C. Brogan, T. Hunter, A. Remijan, J.-C. Guillemin, Millimeter wave Spectroscopy of Cyanoketene $(\mathrm{NC}-\mathrm{CH}=\mathrm{C}=\mathrm{O})$ and its ISM search, Astronomy and Astrophysics accepted.

[41] M. Melosso, A. Melli, C. Puzzarini, C. Codella, L. Spada, L. Dore, C. Degli Esposti, B. Lefloch, R. Bachiller, C. Ceccarelli, J. Cernicharo, V. Barone, Laboratory measurements and astronomical search for cyanomethanimine, Astronomy and Astrophysics 609 (2018) A121-8.

[42] J. Cernicharo, B. Lefloch, M. Agúndez, S. Bailleux, L. Margulès, E. Roueff, R. Bachiller, N. Marcelino, B. Tercero, C. Vastel, E. Caux, Discovery of the Ubiquitous Cation $\mathrm{NS}^{+}$in Space Confirmed by Laboratory Spectroscopy, The Astrophysical Journal Letters 853 (2) (2018) L22.

[43] A. E. Higuchi, N. Sakai, Y. Watanabe, A. López-Sepulcre, K. Yoshida, Y. Oya, M. Imai, Y. Zhang, C. Ceccarelli, B. Lefloch, C. Codella, R. Bachiller, T. Hirota, T. Sakai, S. Yamamoto, Chemical Survey toward Young Stellar Objects in the Perseus Molecular Cloud Complex, The Astrophysical Journal Supplement Series 236 (2) (2018) 52.

[44] B. A. McGuire, P. B. Carroll, N. M. Dollhopf, N. R. Crockett, J. F. Corby, R. A. Loomis, A. M. Burkhardt, C. Shingledecker, G. A. Blake, A. J. Remijan, CSO and CARMA observations of L1157. I. A deep search for hydroxylamine $\left(\mathrm{NH}_{2} \mathrm{OH}\right)$, The Astrophysical Journal 812 (1) (2015) 1-9.

[45] J. K. Jørgensen, F. L. Schöier, E. F. van Dishoeck, Physical structure and CO abundance of low-mass protostellar envelopes, Astronomy and Astrophysics 389 (3) (2002) 908-930.

[46] M. Araki, S. Takano, N. Sakai, S. Yamamoto, T. Oyama, N. Kuze, K. Tsukiyama, Long Carbon Chains in the Warm Carbon-chain-chemistry Source L1527: First Detection of $\mathrm{C}_{7} \mathrm{H}$ in Molecular Clouds, The Astrophysical Journal 847 (1) (2017) 51.

[47] P. Hily-Blant, A. Faure, C. Vastel, V. Magalhaes, B. Lefloch, R. Bachiller, The nitrogen isotopic ratio of $\mathrm{HC}_{3} \mathrm{~N}$ towards the L1544 prestellar core, Monthly Notices of the Royal Astronomical Society 480 (1) (2018) 1174-1186.

[48] A. Crapsi, P. Caselli, C. M. Walmsley, P. C. Myers, M. Tafalla, C. W. Lee, T. L. Bourke, Probing the Evolutionary Status of Starless Cores through $\mathrm{N}_{2} \mathrm{H}$ and $\mathrm{N}_{2}$ D Observations, The Astrophysical Journal 619 (1) (2005) 379-406.

[49] B. A. McGuire, A. M. Burkhardt, S. V. Kalenskii, C. N. Shingledecker, A. J. Remijan, E. Herbst, M. C. McCarthy, Detection of the aromatic molecule benzonitrile $\left(c-\mathrm{C}_{6} \mathrm{H}_{5} \mathrm{CN}\right)$ in the interstellar medium, Science 359 (2018) 202-205.

[50] P. Gratier, L. Majumdar, M. Ohishi, E. Roueff, J. C. Loison, K. M. Hickson, V. Wakelam, A new reference chemical composition for TMC-1, The Astrophysical Journal Supplement Series 225 (2) (2016) 1-10.

[51] B. E. Turner, A molecular line survey of Sagittarius B2 and Orion-KL from 70 to $115 \mathrm{GHz}$. II-Analysis of the data., The Astrophysical Journal Supplement Series 76 (1991) 617-686.

[52] M. J. Frisch, G. W. Trucks, H. B. Schlegel, G. E. Scuseria, M. A. Robb, J. R. Cheeseman, G. Scalmani, V. Barone, B. Mennucci, G. A. Petersson, H. Nakatsuji, M. Caricato, X. Li, H. P. Hratchian, A. F. Izmaylov, J. Bloino, G. Zheng, J. L. Sonnenberg, M. Hada, M. Ehara, K. Toyota, R. Fukuda, J. Hasegawa, M. Ishida, T. Nakajima, Y. Honda, O. Kitao, H. Nakai, T. Vreven, J. A. Montgomery, J. E. Peralta, F. Ogliaro, M. Bearpark, J. J. Heyd, E. Brothers, K. N. Kudin, V. N. Staroverov, R. Kobayashi, J. Normand, K. Raghavachari, A. Rendell, J. C. Burant, S. S. Iyengar, J. Tomasi, M. Cossi, N. Rega, J. M. Millam, M. Klene, J. E. Knox, J. B. Cross, V. Bakken, C. Adamo, J. Jaramillo, R. Gomperts, R. E. Stratmann, O. Yazyev, A. J. Austin, R. Cammi, C. Pomelli, J. W. Ochterski, R. L. Martin, K. Morokuma, V. G. Zakrzewski, G. A. Voth, P. Salvador, J. J. Dannenberg, S. Dapprich, A. D. Daniels, Farkas, J. B. Foresman, J. V. Ortiz, J. Cioslowski, D. J. Fox, Gaussian 09, Revision B.01, Gaussian 09, Revision B.01, Gaussian, Inc., Wallingford CT.

[53] C. Vastel, C. Ceccarelli, B. Lefloch, R. Bachiller, The origin of complex organic molecules in prestellar cores, The Astrophysical Journal Letters 795 (1) (2014) L2-6.

[54] X. Chen, R. Launhardt, T. Henning, IRAM-PdBI observations of binary protostars. I. The hierarchical system SVS 13 in NGC 1333, The Astrophysical Journal 691 (2) (2009) 1729-1737. 
A new study of the $\mathrm{CH}_{3} \mathrm{CHS}$ spectrum up to $660 \mathrm{GHz}$ has been carried out. The rotation transitions in $\mathrm{v}_{\mathrm{t}}=0,1,2$ states with $J$ up to 60 have been assigned. The new data were fit within experimental error using the Rho axis method. Thioacetaldehyde was searched for, but not detected, in the ISM. 


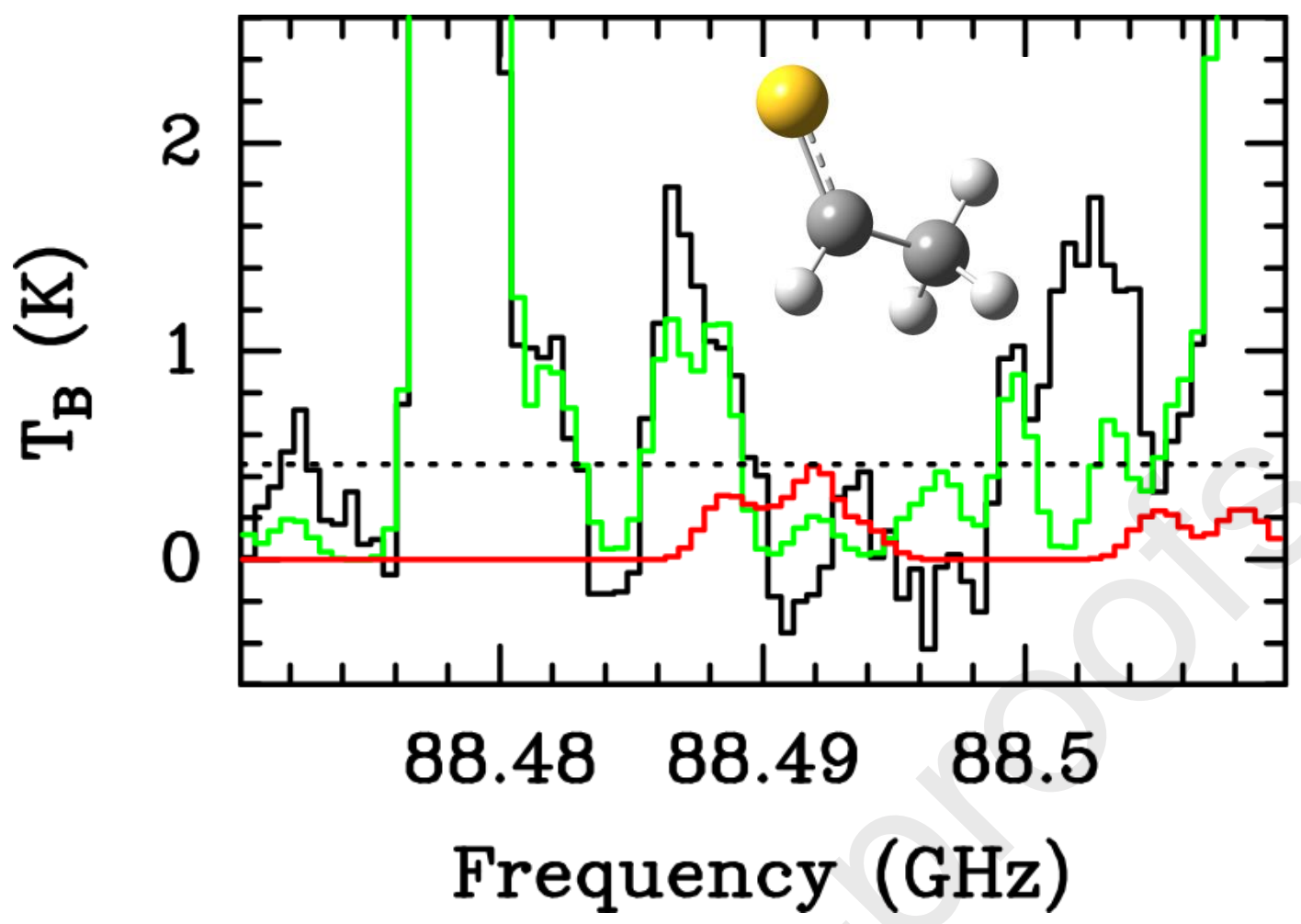


L. Margulès: Conceptualization, Investigation, Formal analysis, Writing - Original Draft V. V. Ilyushin: Formal analysis, Writing - Original Draft

B. A. McGuire: Formal analysis, Writing - Original Draft

A. Belloche: Formal analysis, Writing - Original Draft

R. A. Motiyenko: Investigation, Writing - Review \& Editing

A. Remijan: Formal analysis

E. A. Alekseev: Investigation

O. Dorovskaya: Formal analysis

J.-C. Guillemin: Conceptualization, Investigation, Writing - Original Draft 


\section{Declaration of interests}

$\forall$

$\forall$ The authors declare that they have no known competing financial interests or personal relationships that could have appeared to influence the work reported in this paper.

$\square$ The authors declare the following financial interests/personal relationships which may be considered as potential competing interests: 